\title{
NOTIFICAÇÃO DOS ACIDENTES DE TRABALHO POR EXPOSIÇÃO A MATERIAL BIOLÓGICO ENTRE TRABALHADORAS DA SAÚDE
}

\author{
Amanda Laís de Oliveira de Moraes*, Adriana Saraiva Aragão dos Santos**, \\ Kionna Oliveira Bernardes*** \\ Autor correspondente: Amanda Lais de Oliveira de Moraes - amandafisio.rj@gmail.com \\ * Estudante de graduação em Fisioterapia, Universidade Federal da Bahia \\ ** Fisioterapeuta. Doutora em Ciências da Saúde pela FCMSCSP, Docente da Universidade Federal da Bahia, \\ Departamento de Biofunção \\ *** Fisioterapeuta. Doutora em Saúde Pública pelo ISC/UFBA, Docente da Universidade Federal da Bahia, Departamento \\ de Biofunção
}

\section{Resumo}

Objetivo: Descrever a ocorrência de acidente de trabalho por material biológico entre trabalhadoras da saúde no Brasil em 2014. Métodos: Estudo de casuística das notificações de trabalho por material biológico em mulheres disponibilizadas pelo Sistema de Informação de Agravos de Notificação em 2014. Foram calculadas frequências absolutas e relativas. Resultados: Foram notificados 22.361casos em todo território nacional, $48,8 \%$ no Sudeste, $23 \%$ no Sul, $15,3 \%$ no Noredeste, $6,8 \%$ no Centro Oeste e $6,1 \%$ no Norte. O perfil encontrado foi mulheres brancas de 31 a 45 anos, com ensino médio e 2,3\% gestantes. A maioria possuía vínculo estável e tinham de 1 a 5 anos de trabalho. A exposição caracterizou-se percutânea, por sangue através de administração de medicação, o agente principal foi agulha com lúmen e $71,42 \%$ eram trabalhadoras de emergências móveis. Apenas a luva aparece com boa adesão. Para o anti-HBS 29,1\% dos exames foram positivos e $20,8 \%$ foram negativos, $93,1 \%$ vacinadas, $62,1 \%$ evoluíram com alta de paciente fonte negativo e $78 \%$ emitiram a comunicação de acidente de trabalho. Conclusão: A notificação ainda não é realidade no Brasil e precisa ser incentivada. Os achados permitem comparar o percentual de notificações por região do Brasil e descrever a ocorrência desses acidentes. Espera-se que o estudo contribua para alertar e informar profissionais e instituições e para estimular o investimento em ações de biossegurança.

Palavras-chave: Saúde do Trabalhador; Epidemiologia; Risco Ocupacional; Sistemas de Informação em Saúde; Saúde da Mulher. 


\title{
NOTIFICATION OF OCCUPATIONAL ACCIDENTS \\ BY EXPOSURE TO BIOLOGICAL MATERIAL AMONG \\ HEALTH WORKERS
}

\begin{abstract}
Objective: To describe the occurrence of work-related accidents caused by biological material among health care providers in Brazil in 2014. Methods: A case study of female work-related notifications by biological material, found in the Information System for Notifiable Diseases in 2014. Absolute and relative frequencies were calculated. Results: 22,361 cases were recorded, of which $48.8 \%$ happened in the Brazilian South East area. The profile found comprised of white women ranging from 31 to 45 years old, with major degree of high school and $2.3 \%$ were pregnant at the time of the accident. Most of them were legally employed and stable at their positions, and had been working with that particular employer from 1 to 5 years. The exposure to the biological material was characteristically percutaneous, by blood through medication management, and the main contamination agent was needle with lumen. Indeed, $71.42 \%$ were working of mobile emergency units. The glove was the only material wich appeared to have good adhesion by the employers. For the anti-HBS, $29.1 \%$ of the exams were positive and $20.8 \%$ were negative. $93.1 \%$ were vaccinated, $62.1 \%$ were discharged because their test results came back negative, and $78 \%$ issued the work-related notification. Conclusion: Work-related Notification is not a reality fully employed tool among health workers in Brazil and it needs to be encouraged. The present findings allow for a comparison of the percentage of notifications per area and a description of the occurrence of these accidents. It is hoped that this study will be helpful to alert and to inform professionals and institutions and to stimulate investments in biosecurity actions.
\end{abstract}

Kepwords: Occupational Health; Epidemiology; Occupational Risks; Health Information Systems; Women's Health.

\section{INTRODUÇÃO}

Os acidentes de trabalho possuem alta incidência de acordo com dados do Ministério do Trabalho e Emprego (MTE), porém ainda não existe uma real estimativa nacional do número de trabalhadores acidentados e suas consequências. Essa subnotificação acontece pela falta de registro e subenumeração desses acidentes nos estabelecimentos de saúde, o que dificulta o planejamento e a adoção de

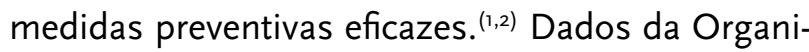
zação Mundial da Saúde (OMS) referem que, dos agravos relacionados à saúde do trabalhador, apenas 1\% a $4 \%$ são notificados na América Latina. (3)
No Brasil a Norma Regulamentadora 32 (NR-32) foi criada a partir do elevado número de acidentes de trabalho entre trabalhadores de saúde, o absenteísmo e os custos do tratamento profilático, para os acidentados, chamou a atenção do MTE. Para a prevenção de acidentes e doenças ocupacionais entre profissionais da saúde, a NR-32 representa a principal normatização brasileira e possui três grandes eixos. O primeiro é a capacitação contínua dos trabalhadores, o segundo define os programas que tratam dos riscos e, por fim, o terceiro eixo determina as medidas de proteção contra os riscos ocupacionais. ${ }^{(4,5)}$ 
Os riscos ocupacionais são classificados em biológicos, físicos, químicos, mecânicos, fisiológicos, ergonômicos e psíquicos. ${ }^{(6)}$ Reconhecer o risco significa identificar, no ambiente de trabalho, fatores ou situações com potencial dano à saúde do trabalhador. ${ }^{(7)}$ Existem evidências de que o trabalhador da área de saúde está constantemente sujeito a riscos ocupacionais de diversas origens, ressaltando-se o biológico, uma vez que se expõem diariamente ao contato com sangue e outros fluidos orgânicos contaminados por uma variedade imensa de patógenos desencadeadores de doenças, e que três delas são de grande relevância: a Síndrome da Imunodeficiência Adquirida (AIDS), a Hepatite B e a Hepatite C. ${ }^{(8)}$

As exposições ocupacionais a materiais biológicos potencialmente contaminados, dependendo da gravidade e das condições do hospedeiro, podem trazer consequências como a redução permanente ou temporária da capacidade para o exercício da profissão, necessidade de afastamento de suas atividades, absenteísmo, necessidade de reorganização do trabalho para dar continuidade ao cuidado e ainda prejuízos financeiros para a instituição. $(9,10)$

Para iniciar o desenvolvimento de ações preventivas e a correta tomada de decisão os Acidentes de Trabalho com Exposição a Material Biológico (ATBIO) devem ser notificados. Entretanto sabe-se que ainda existe um elevado índice de subnotificação em decorrência da falta de informação quanto à esta necessidade e também devido ao receio de demissão ou críticas por parte do acidentado, o que o leva a não notificar os ATBIO. ${ }^{(10)}$

O ATBIO, representado pelo CID Z20.9, é considerado agravo de notificação compulsória. O Ministério da Saúde possui uma ficha padronizada para notificação de ATBIO pelo Sistema de Informação de Agravos de Notificação (SINAN) que é um sistema de coleta e processamento de dados que transmite e dissemina informações sobre doenças e agravos de notificação compulsória. Através do preenchimento da ficha as informações são registradas possibilitando conhecer o perfil epidemiológico a nível municipal, estadual e federal. ${ }^{(11)}$
Em 2010 o SINAN notificou 32.734 casos de AT$\mathrm{BIO}$, onde 24.540 atingiram o sexo feminino. ${ }^{(12)}$ Isso pode ser explicado pela maior dominância de mulheres nas profissões da saúde decorrente do aumento do nível de escolaridade e facilidade de acesso às universidades capacitando, assim, a busca a postos de melhor remuneração. ${ }^{(13,14)}$

Sendo assim, conhecer a incidência do ATBIO no Brasil contribui para aumentar a compreensão sobre os processos determinantes para a ocorrência dos acidentes a fim de gerar subsídios para novas pesquisas e programas de intervenção. Logo, o objetivo desse estudo foi descrever a ocorrência de acidente de trabalho com exposição potencial a material biológico entre trabalhadoras da saúde no Brasil no ano de 2014.

\section{MATERIAL E MÉTODOS}

Foi realizada uma pesquisa descritiva com abordagem quantitativa sobre a distribuição dos casos de acidente de trabalho com exposição a material biológico, envolvendo trabalhadoras da saúde, com os dados nacionais do ano de 2014.

Foram utilizados dados secundários de uma fonte de pesquisa proveniente do DATASUS, com base no Sistema de Informação de Agravos de Notificação (SINAN/DATASUS) disponibilizados pelo Centro Colaborador de Vigilância aos Acidentes de Trabalho (CCVISAT) em www.ccvisat.ufba.br. foram avaliadas todas as notificações com CID Z20.9 (acidente de trabalho com exposição à material biológico) informados nas fichas de notificação.

As variáveis de interesse utilizadas foram: características sócio-demográficas: idade, condição de gestação, raça/cor, escolaridade, região; característica da ocupação: Situação no mercado de trabaIho, tempo de trabalho na ocupação, Atividade econômica CNAE; caracterização do acidente: tipo de exposição, material orgânico, circunstância do acidente, agente, uso de EPI; desfecho do caso: situação vacinal do acidentado em relação à hepatite $B$, resultados de exames do acidentado, conduta no 
momento do acidente, evolução do caso, foi emitida a comunicação de acidente de trabalho.

A análise dos dados foi feita através do Programa Statistical Products and Service Solutions (SPSS) 22, onde foram calculadas frequências absolutas e relativas para descrição das variáveis de interesse, além de média e desvio padrão para a variável "tempo de trabalho". Foi confeccionado um gráfico com auxílio do programa Excel 2010. A figura se encontra disponível no site www.mapasparacolorir.com.br. Por se tratar de pesquisa de fonte secundária, o estudo não foi submetido a um Comitê de Ética e Pesquisa, entretanto atendeu a resolução vigente $n^{\circ} 466$ do Conselho Nacional de Saúde de 2015.

\section{RESULTADOS E DISCUSSÃO}

No ano de 2014 O SINAN registrou 22.361 casos de ATBIO em mulheres no Brasil. Diversos estudos apontam para a feminilização das profissões da saúde, o que repercute em altos índices de ATBIO nessa população. ${ }^{(14)} A$ frequência de notificação por região (figura 1), distribui-se de modo heterogêneo, registrou-se $48,8 \%$ dos ATBIO no Sudeste, seguidas pelas regiões Sul, Nordeste, Centro-Oeste e Norte, com $23 \%, 15,3 \%, 6,8 \%$ e $6,1 \%$ respectivamente. O maior contingente de estabelecimentos de saúde nas regiões sul e sudeste e a consequente concentração de mão de obra podem justificar o volume de notificações de ATBIO. Além disso, a vigilância em saúde do trabalhador, nestas regiões encontra se consolidadas

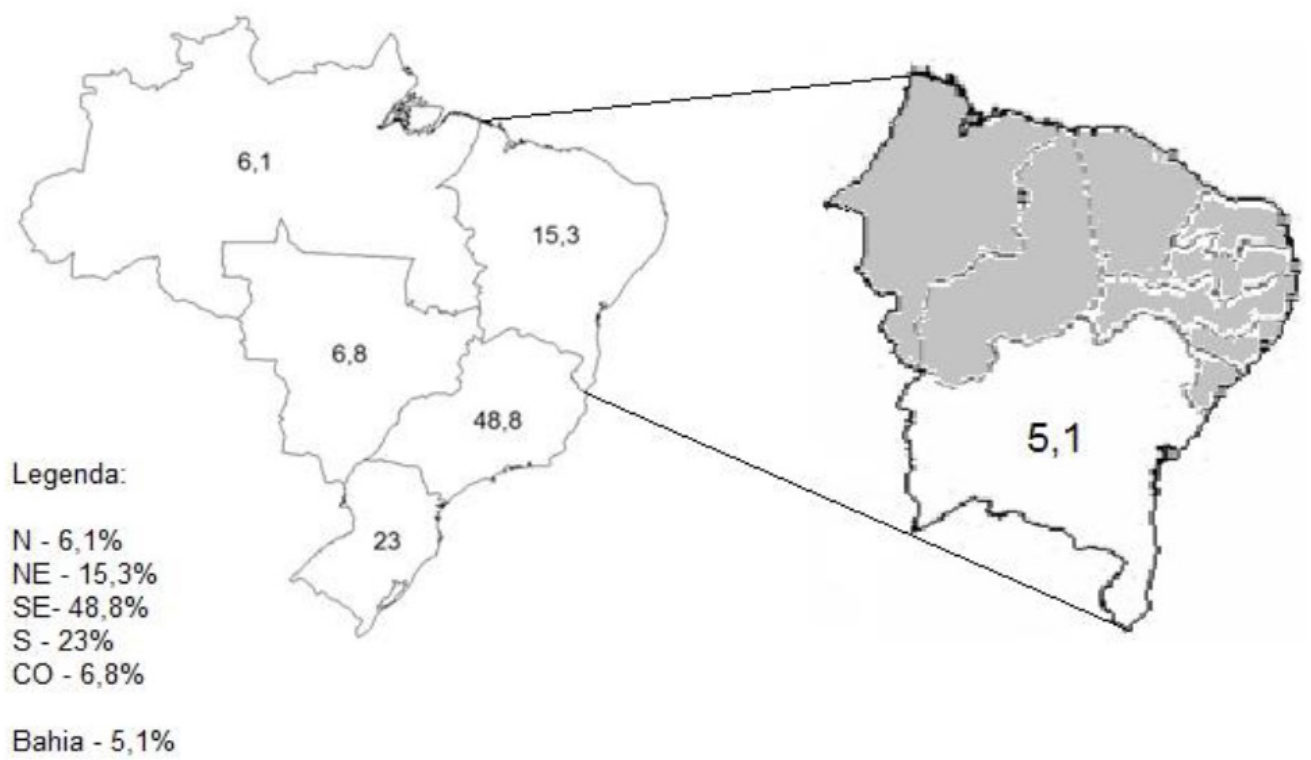

Figura 1 - Distribuição das notificações de ATBIO em mulheres por região do Brasil,no ano de 2014 com destaque para - Estado da Bahia (valor percentual).

Fonte: DATASUS/SINAN - 2014

O percentual do Nordeste apresentou $15,3 \%$ de ATBIO em mulheres no ano de 2014 , com 5,1\% referentes apenas ao Estado da Bahia. Considerando as 9 Unidades Federativas (UFs) da região é provável a subnotificação existente nos demais Estados. Vale ressaltar também que o percentual da Bahia é alto quando comparado a regiões inteiras como o Centro-Oeste e o Norte que aparecem com 6,8 e $6,1 \%$. No entanto, a subnotificação é provocada principalmente pela não valorização das pequenas lesões e pela pouca estrutura das unidades destinadas a este atendimento específico. ${ }^{(15)} \mathrm{Não}$ há na 
literatura estudos que comparem o percentual de notificações por região no Brasil.

O perfil sóciodemográfico encontrado nesse estudo caracteriza-se por mulheres brancas $62,4 \%$, de 31 a 45 anos $43,2 \%$, com escolaridade de ensino médio $58,4 \%$ e poucas gestantes $2,3 \%$ (Tabela 1 ). Os achados desta pesquisa referente à raça/cor corroboram com os demais estudos. Para a variável faixa etária, os resultados observados na litera- tura não são unânimes, pois alguns demonstram que a exposição é mais frequente em pessoas mais jovens, entre 19 e 30 anos e outros referem entre 30 e 40 anos, todos eles incluem o sexo masculino. ${ }^{(12,15,16)}$ Os estudos que utilizam a variável nível de escolaridade encontraram o mesmo resultado deste. ${ }^{(12,16)}$ Atualmente esta variável tem sido substituída por "categoria profissional" em diversos trabalhos. Não foi encontrado nenhum trabalho que analisou a ocorrência de ATBIO em gestantes.

Tabela 1 - Perfil sociodemográfico das notificações de ATBIO,em mulheres no Brasil em 2014

\begin{tabular}{lcc}
\hline \multicolumn{1}{c}{ CaRACTERÍsticas } & N & $\%$ \\
\hline Raça/Cor (20.528) & 12.814 & \\
Branca & 7.714 & 62,4 \\
Não branca & & 37,6 \\
Faixa etária (22.159) & 9.134 & \\
15 a 30 anos & 9.578 & 41,2 \\
31 a 45 anos & 3.163 & 43,2 \\
46 a 59 anos & 284 & 14,3 \\
Acima de 6O anos & & 1,3 \\
Escolaridade (18.452) & 14 & 0,1 \\
Analfabeta & 1.248 & 6,7 \\
Ensino Fundamental & 10.769 & 58,4 \\
Ensino Médio & 6.421 & 34,8 \\
Ensino Superior & & 17.831 \\
Condição de gestação (18.255) & 424 & 97,7 \\
Gestante & 2,3 & \\
Não gestante & &
\end{tabular}

Fonte: DATASUS/SINAN - 2014.

No que se refere às características da ocupação (tabela 2), foi avaliado a situação no mercado de trabalho e o tempo de trabalho. Engloba formal a trabalhadora registrada com carteira assinada e servidoras públicas, que predominaram entre as notificações com 89,5\%. Nesse contexto, a maior exposição de ATBIO esteve presente entre trabaIhadoras que referiram até 5 anos de tempo de trabalho na ocupação, com $43,8 \%$, assim a média do tempo de trabalho foi 5,39 anos e o desvio padrão foi 7,68 . No estudo de Valim et al ${ }^{(15)}$ também foi encontrado até cinco anos de tempo de trabalho. Contudo, não há um consenso na literatura da relação entre experiência profissional e ocorrência de ATBIO. (17) Profissionais com menor tempo de atuação, apesar de evidenciarem uma atividade profissional em consolidação, podem estar mais dispostos a adotarem as medidas de precaução padrão do que o profissional que exerce suas atividades há mais tempo. Sabe-se que o maior tempo de trabalho não evita nem reduz o risco de ocorrência de ATBIO. ${ }^{(10,18)}$ 
Tabela 2 - Características da ocupação dos casos notificados com ATBIO, em mulheres no Brasil em 2014

\begin{tabular}{lcc}
\hline \multicolumn{1}{c}{ CaRACTERísticas } & N & $\%$ \\
\hline Situação no mercado (18.388) & 16.459 & \\
Formal & 1.912 & 89,5 \\
Informal & 17 & 10,4 \\
Aposentada & & 0,1 \\
Tempo de Trabalho (22.361) & 5.412 & \\
Menos de 1 ano & 9.803 & 24,20 \\
De 1 a 5 anos & 3.878 & 43,83 \\
De 6 a 1O anos & 2.242 & 17,34 \\
De 11 a 2O anos & 1.026 & 10,02 \\
Acima de 21 anos & 4,61 \\
\hline
\end{tabular}

Fonte: SINAN/DATASUS - 2014.

Foi feito um levantamento, utilizando-se a Classificação Nacional de Atividade Econômica (CNAE), das atividades que registraram o maior número de ATBIO em mulheres no ano em estudo (gráfico 1). Sobre o percentual de acometimento, observa-se que as trabalhadoras de emergências móveis disparam em primeiro lugar nos ATBIO com $71,42 \%$, em seguida aparecem as profissionais da clínica de saúde com 9,81\% e do serviço laboratorial com $9,36 \%$, nessa ordem. Considera-se que essa é uma importante categoria, mas o número de preenchi- mento ainda aparece baixo ou ausente na ficha de notificação, pois de 22.361 casos, apenas 3.894 preencheram a CNAE. Pesquisas mostram que trabalhadores de emergências móveis possuem contato maior e mais intenso com o paciente e estão sujeitos a fatores agravantes como realizar o atendimento fora do ambiente hospitalar em condições quase sempre inadequadas, além das características inerentes ao atendimento pré-hospitalar móvel: rapidez, estresse, equipamentos insuficientes ou em estado precário de conservação, insegurança e violência, dentre outros. ${ }^{(1,19)}$

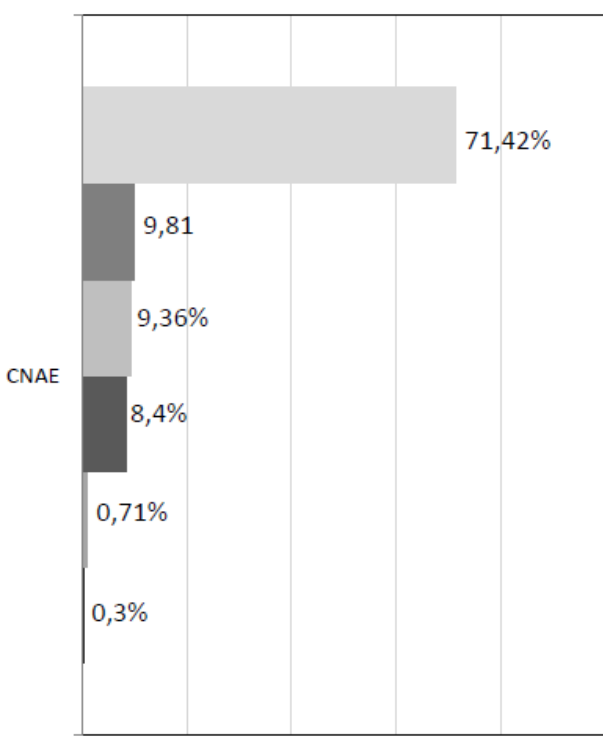

Emergências móveis

Clínica de saúde

Serviço laboratorial

Terapias alternativas

Equipe multidisciplinar da

saúde

- Hospitalar

Gráfico 1 - Representação da CNAE nos casos de ATBIO em mulheres no Brasil em 2014. 
O ATBIO pode trazer consequências para o profissional como: infecção, contaminação, perda de benefícios trabalhistas, redução salarial, transferência de setor e insegurança devido ao resultado dos exames, além de problemas familiares, que por sua vez, interferem na produção, aumentam o custo operacional e reduzem a eficiência no trabalho. ${ }^{(9)}$ A prevenção depende, em parte, da identificação dos riscos, portanto uma boa estratégia preventiva seria a realização de cursos de atualização em biossegurança a todos os profissionais atuantes na área da saúde ou a implementação de programas de educação continuada, principalmente em ambientes hospitalares com o objetivo de aumentar o conhecimento sobre as medidas de precauções-padrão, bem como incentivar os profissionais a refletirem sobre sua prática, biossegurança e responsabilidade social. ${ }^{(20,21)}$
No presente estudo, o acidente se caracterizou por exposição percutânea, representando 79,5\% dos casos. Percebe-se que, para a circunstância do acidente, 21,5\% aconteceu através de administração de medicação, onde o agente principal foi a agulha com lúmem $62,5 \%$, sendo $84,8 \%$ através do sangue (tabela 3). A descrição das características do acidente revela fatores de exposição para o desenvolvimento de ATBIO. O estudo de Ronald et al ${ }^{(14)}$ objetivou verificar a prevalência das notificações de acidentes envolvendo material biológico que acometeram profissionais da saúde, apresentando semelhanças com o presente estudo em relação ao tipo de exposição, ao material orgânico e ao agente. Em outras pesquisas também prevalece o sangue como material orgânico mais encontrado ${ }^{(20,22,23)}$ mesmo após exposição ao sangue alguns acidentados não procuram atendimento clínico especializado. ${ }^{(16)}$

Tabela 3 - Caracterização do ATBIO em mulheres no Brasil em 2014

(continua)

$\begin{array}{lll}\text { CARACTERÍSTICAS } & \mathrm{N} & \%\end{array}$

Tipo de exposição Percutânea (20.958)

Sim

Não

Pele íntegra (19.077)

Sim

Não

Pele não-íntegra (18.519)

Sim

Não

17.398

13,6

Sim

16.334

86,4

Outros (16.614)

Sim

Não

16.345

17.125

3.077

79,5

20,5

31,6

68,4

93,9

98,4

Material orgânico (20.202)

Sangue

Outros 
Tabela 3 - Caracterização do ATBIO em mulheres no Brasil em 2014

\begin{tabular}{|c|c|c|}
\hline CaRACTERÍSticas & $N$ & $\%$ \\
\hline \multicolumn{3}{|l|}{ Agente (21.306) } \\
\hline Agulha com lúmen & 13.317 & 62,5 \\
\hline Agulha sem lúmen & 1.926 & 9,0 \\
\hline Intracath & 160 & 0,8 \\
\hline Vidros & 182 & 0,9 \\
\hline Lâminas & 1.586 & 7,4 \\
\hline Outros & 4.135 & 19,4 \\
\hline \multicolumn{3}{|l|}{ Circunstância do acidente (21.627) } \\
\hline Administração de medicação & 4.643 & 21,5 \\
\hline Punção & 2.508 & 11,6 \\
\hline Descarte inadequado & 3.460 & 16 \\
\hline Lavanderia & 146 & 0,7 \\
\hline Lavagem de material & 897 & 4,1 \\
\hline Manipulação de caixa com material & 1.224 & 5,7 \\
\hline Procedimento cirúrgico & 1.588 & 7,3 \\
\hline Procedimento odontológico & 1.018 & 4,7 \\
\hline Procedimento laboratorial & 518 & 2,4 \\
\hline Dextro & 1.027 & 4,7 \\
\hline Reencape & 636 & 2,9 \\
\hline Outros & 3.962 & 18,4 \\
\hline \multicolumn{3}{|l|}{ Uso de EPI Luva (20.823) } \\
\hline Sim & 15.647 & 75,1 \\
\hline Não & 5.176 & 24,9 \\
\hline \multicolumn{3}{|l|}{ Avental (19.662) } \\
\hline Sim & 8.706 & 44,3 \\
\hline Não & 10.956 & 55,7 \\
\hline \multicolumn{3}{|l|}{ Óculos (19.494) } \\
\hline Sim & 3.263 & 16,7 \\
\hline Não & 16.231 & 83,3 \\
\hline \multicolumn{3}{|l|}{ Máscara (19.515) } \\
\hline $\operatorname{Sim}$ & 4.916 & 25,2 \\
\hline Não & 14.599 & 74,8 \\
\hline \multicolumn{3}{|l|}{ Proteção facial (19.043) } \\
\hline Sim & 917 & 4,8 \\
\hline Não & 18.126 & 95,2 \\
\hline \multicolumn{3}{|l|}{ Bota (18.964) } \\
\hline Sim & 2.395 & 12,6 \\
\hline Não & 16.569 & 87,4 \\
\hline
\end{tabular}

Fonte: SINAN/DATASUS -2014. 
Com a finalidade de reduzir a exposição do trabalhador, os empregadores são obrigados a fornecer os EPI's adequados ao risco aos quais o profissional se expõe. O dispositivo é de uso individual e inclui luvas, protetores oculares ou faciais, protetores respiratórios, aventais e proteção para os membros inferiores. Deve-se ultrapassar barreiras como a autoconfiança, o descuido e a pressa para o uso eficaz dos EPI's. ${ }^{(21,24)}$ Assim como em outros estudos, observou-se que no momento do acidente os profissionais não utilizavam todos os equipamentos de proteção, o que contribui para a ocorrência de ATBIO. ${ }^{(14,15)}$ Dentre as acidentadas $75,1 \%$ utilizaram luvas, porém ainda é necessária maior aceitação dos profissionais aos demais componentes como o avental $44,3 \%$, o óculos $16,7 \%$, a máscara $25,2 \%$, a proteção facial $4,8 \%$ e a bota $12,6 \%$.

Dentre os resultados dos exames dos casos analisados (tabela 4 ) $0,6 \%$ deram positivo para
anti-HIV e para HbsAg, 29,1\% sinalizaram positivo para anti-HBS, 20,8\% foram negativos para anti-HBS, ou seja, com suspeita do vírus, 1,8\% foram inconclusivos e $22,5 \%$ não realizados para esse exame. Para anti-HCV $0,4 \%$ deram positivo. O percentual do risco de contaminação pelo vírus da Hepatite $B$ encontrado nesse trabalho foi de $20,8 \%$, essa taxa é similar a de outros estudos que comprovam que esse risco varia de $6 \%$ a $30 \% . .^{(16,23)}$ Verifica-se que $93,1 \%$ das acidentadas eram vacinadas, $74,9 \%$ tiveram indicação de quimioprofilaxia, $1,6 \%$ se recusaram a quimioprofilaxia indicada. Algumas se submeteram a terapias antirretrovirais como a $\mathrm{AZT}+3 \mathrm{TC}(14,5 \%), \mathrm{AZT}+3 \mathrm{TC}$ Indinavir (1,8\%), AZT+3TC Nelfinavir (0,5\%), Imunoglobulina humana contra hepatite $B$ (HBIG) 3,8\% e ainda $7,7 \%$ foram vacinadas contra Hepatite B. Evoluíram com alta de paciente fonte negativo $62,1 \%$ (tabela 5).

Tabela 4 - Resultado dos exames nos casos de ATBIO em mulheres no Brasil em 2014

(continua)

\begin{tabular}{lcc}
\hline \multicolumn{1}{c}{ Resultado dos EXAMES } & N & $\%$ \\
\hline Anti HIV (20.116) & 115 & 0,6 \\
Positivo & 13.012 & 64,7 \\
Negativo & 253 & 1,3 \\
Não concluído & 2.324 & 11,6 \\
Não realizado & 4.412 & 21,8 \\
Não informado & & \\
HbsAg (19.914) & 111 & 0,6 \\
Positivo & 10.568 & 53,1 \\
Negativo & 320 & 1,6 \\
Não concluído & 3.869 & 19,4 \\
Não realizado & 5.046 & 25,3 \\
Não informado & & \\
Anti HBS (19.844) & 5.776 & 29,1 \\
Positivo & 4.123 & 20,8 \\
Negativo & 350 & 1,8 \\
Não concluído & 4.460 & 22,5 \\
Não realizado & 5.135 & 25,8 \\
Não informado & &
\end{tabular}


Tabela 4 - Resultado dos exames nos casos de ATBIO em mulheres no Brasil em 2014

\begin{tabular}{lcc}
\hline Resultado dos EXAMES & N & $\%$ \\
\hline Anti HCV (19.917) & 80 & 0,4 \\
Positivo & 11.202 & 56,2 \\
Negativo & 336 & 1,7 \\
Não concluído & 3.251 & 16,3 \\
Não realizado & 5.048 & 25,4 \\
Não informado & & \\
\hline
\end{tabular}

Fonte: SINAN/DATASUS -2014

Tabela 5 - Conclusão dos casos de ATBIO em mulheres no Brasil em 2014

(continua)

\section{Características}

Situação vacinal (20.190)

Vacinada

18.802

Não vacinada

1.388

\section{Condutas Sem indicação de}

quimioprofilaxia (17.247)

Sim

12.912

Não

4.355

227

13.758

2.087

12.313

258

Não

13.764

AZT+3TC+Nelfinavir (13.932)

Sim

Não

13.859

539

13.489

Sim

Não

1.084

Sim

13.075
93,1

6,9

74,9

1,8

98,2

0,5

3,8

7,7

$\%$

25,1

1,6

98,4

14,5

85,5

99,5

96,2

92,3 
Tabela 5 - Conclusão dos casos de ATBIO em mulheres no Brasil em 2014

(conclusão)

\begin{tabular}{lcc}
\hline \multicolumn{1}{c}{ CARACTERísticAS } & N & $\%$ \\
\hline Outro esquema ARV (14.126) & 1.723 & 12,2 \\
Sim & 12.403 & 87,8 \\
Não & & \\
Evolução do caso (10.036) & 210 & 2,1 \\
Alta com conversão & 2.829 & 28,2 \\
Alta sem conversão & 6.230 & 62,1 \\
Alta fonte negativa & 763 & 7,6 \\
Abandono & 1 & 0,0 \\
Óbito por ATBIO & 3 & 0,0 \\
Óbito por outra causa & & 78 \\
Emissão a CAT (14.183) & 11.066 & 22 \\
Sim & 3.117 & \\
Não & & 78 \\
\hline
\end{tabular}

Fonte: SINAN/DATASUS -2014.

Mesmo com a maioria vacinada previamente, apenas $29,1 \%$ apresentaram resultado reagente para o anticorpo anti-HBs, o que indica que as profissionais não estão totalmente protegidas contra o vírus da Hepatite $\mathrm{B}$, ou por não completar o esquema vacinal ou por não apresentar resposta vacinal. Nos estudos de Silva et $\mathrm{al}^{(20)}$ e de Valim et al ${ }^{(15,20)}$ também não foi encontrada resposta reagente ao anti-HBS ao comparar a taxa de imunização com os resultados dos exames. ${ }^{(20)} A$ vacinação dos trabalhadores da saúde minimiza a incidência de infecção em $95 \%$ e é considerada como uma das medidas preventivas mais importantes para a prevenção desse vírus. ${ }^{(15,23)}$

Em caso de exposição o cuidado imediato consiste em lavar bem o local com água e sabão ou soro fisiológico $0,9 \%$ nas mucosas. Porém, em casos mais graves, de acordo com critérios individuais, são necessárias condutas mais específicas e que requerem certos limites de tempo para seres executadas, como no caso de profilaxia das infecções pelo HIV e Hepatite B. Nestes casos, a imunização é essencial, mas apenas minimiza a gravidade do acidente. ${ }^{(10,20)}$
Constatou-se ainda que $78 \%$ emitiram a CAT. O profissional que presta atendimento à vítima, é responsável por preencher e assinar a CAT. Há necessidade de solicitar exames sorológicos conforme orientação nacional e internacional, portanto o responsável deve estar preparado para avaliar o acidentado.(1)

Notificar o acidente é importante para o investimento em ações preventivas e educativas a fim de conscientizar as trabalhadoras, o que pode contribuir para diminuir a incidência e melhorar as condutas pós-exposição.

Como limitação do estudo, destaca-se que não foram calculados coeficientes de incidência. Outro aspecto importante a ser observado, é a subnotificação que não permite quantificar a realidade nacional dos ATBIO.

\section{CONCLUSÃO}

Considerando os riscos de exposição dessa população, os resultados deste trabalho e os dados da literatura percebe-se que a notificação de ATBIO 
ainda não é realidade no Brasil e precisa ser incentivada através de pesquisas e da prática de biossegurança nas instituições de saúde.

Os achados são importantes porque permitem comparar o percentual de notificações por região do Brasil, bem como descrever a ocorrência desses acidentes nas profissionais da saúde, que é a população com a maior incidência de ATBIO.

As trabalhadoras da saúde notificadas por ATBIO em 2014 caracterizam-se por mulheres brancas de 31 a 45 anos, com escolaridade de ensino médio e evoluem com exposição percutânea, sendo o sangue o fluido mais notificado através de administração de medicação com agulha com lúmen A maioria dos ATBIO acontecem em trabalhadoras de emergências móveis. A adesão ao uso de EPI foi baixa, apenas a luva está, de fato, inserida na proteção do profissional. As mulheres evoluíram com alta de paciente fonte negativo e a quantidade de emissão a CAT aumentou cerca de $48,2 \%$ em comparação a outros estudos.

Sugere-se estudar se existe diferença na ocorrência de ATBIO por região do Brasil, para que se conheçam as dificuldades mais específicas enfrentadas e a partir disso se definam estratégias preventivas adequadas a cada região. Espera-se que o presente estudo contribua para alertar e informar profissionais e instituições quanto a importância da notificação dos acidentes e para estimular o investimento em ações de biossegurança.

\section{REFERÊNCIAS}

1. Paiva MHRS, Oliveira AC. Fatores determinantes e condutas pós-acidente com material biológico entre profissionais do atentimento pré-hospitalar. Rev Bras Enferm. 2011; 64(2):268-73.

2. Gusmão GS, Oliveira AC, Gama CS. Acidente de trabalho com material biológico: análise da ocorrência e do registro. Cogitare Enferm. 2013;18(3):558-64.

3. Secretaria Estadual de Saúde (Rio Grande do Sul). [Acesso em 2015 abr 25].
Disponível em: http://www.saude.rs.gov. $\mathrm{br} /$ conteudo/7129/?Sa\%C3\%BAde_do_ trabalhador_no_s\%C3\%A9culo_21_\%C3\%A9. tema_de_semin\%C3\%Alrio

4. Marziale MHP, Rocha FLR, Robazzi MLCC, Cenzi CM, Santos HEC, Trovó MEM. Influência organizacional na ocorrência de acidentes de trabalho com exposição a material biológico. Rev Lat Am Enferm. 2013;21(8).

5. Ministério do Trabalho e Emprego (BR). Portaria $\mathrm{n}^{\circ} 485$, de 11 de novembro de 2005. [acesso em 2014 out O6]. Disponível em: http://portal.mte. gov.br/legislacao/portaria-n-485-de-11-11-2005. htm

6. Sêcco $\mid A O$, Robazzi MLCC, Shimizu DS, Rúbio MMS. Acidentes de Trabalho Típicos Envolvendo Trabalhadores de Hospital Universitário da Região Sul do Brasil: Epidemiologia e Prevenção. Rev. Lat Am Enferm. 2008;16(5).

7. Ministério da Saúde (BR). Os riscos biológicos no âmbito da Norma Regulamentadora 32. Brasília; 2008.

8. Silva MR, Cortez EA, Valente GSC. Acidentes com materiais perfurocortantes e biológicos no ambiente hospitalar: análise da exposição ao risco e medidas preventivas. Rev Pesqui Cuid Fundam. 2011;3(2):1856-72.

9. Claudio CV, Sarquis LMM, Scussiato LA, Miranda FMD. Monitoramento biológico sob a ótica dos enfermeiros gerentes. Rev Rene. 2013;14(2):252-61.

10. Araújo TM, Caetano JA; Barros LM, Lima ACF, Costa RM, Monteiro VA. Acidentes de trabalho com exposição a material biológico entre os profissionais de Enfermagem. Rev. Enferm. Ref. 2012;3(7):7-14.

11. Ministério da Saúde (BR). Portaria n ${ }^{\circ}$ 1.271, de 6 de junho de 2014 [acesso em 2015 abr. 25]. Define a Lista Nacional de Notificação Compulsória de doenças, agravos e eventos de saúde pública nos serviços de saúde públicos e privados em todo o território nacional, nos termos do anexo, e dá outras providências. Disponível em: http://bvsms.saude.gov.br/bvs/ saudelegis/gm/2014/prt1271_O6_O6_2O14.html

12. Acidentes de Trabalho com Exposição Potencial a Material Biológico. Boletim Epidemiológico. 
Informe do Centro Colaborador UFBA/ISC/ PISAT - MS/DSAST/CGSAT. 2011;3(1).

13. Spagnuolo RS, Baldo RCS, Guerrini IA. Análise epidemiológica dos acidentes com material biológico registrados no Centro de Referência em Saúde do Trabalhador. Rev Bras Epidemiol. 2008;11(2):315-23.

14. Martins RJ, Moimaz SAS, Garbin AJI, Gonçalves PRV, Garbin CAS. Prevalência de Acidentes com Material Biológico em um Município do Noroeste de São Paulo, Brasil, no Período de 2007 a 2011. Ciência e Trabalho. 2014;16(50):93-96.

15. Valim MD, Marziale MHP, Hayashida M, Martínez MR. Ocorrência de acidentes de trabalho com material biológico potencialmente contaminado em enfermeiros. Acta Paul Enferm. 2014;27(3):280-6.

16. Pimenta FR, Ferreira MD, Gir E, Hayashida M, Silvia RMS, Canini SRMS. Atendimento e seguimento clínico especializado de profissionais de enfermagem acidentados com material biológico. Rev Esc Enferm. 2013;47(1):198-204.

17. Câmara PF, Lira C, Junior BJS, Vilella TAS, Hinrichsen SL. Investigação de acidentes biológicos entre profissionais da equipe multidisciplinar de um hospital. Rev Enferm UERJ. 2011;19(4):583-6.

18. Oliveira AC, Paiva MHRS. Análise dos acidentes ocupacionais com material biológico entre profissionais em serviços de atendimento préhospitalar. Rev Lat Am Enferm. 2013. 21(1):7.

19. Paiva MHRS. Acidentes ocupacionais por exposição a materiais biológicos entre trabalhadores do serviço de atendimento pré-hospitalar móvel de minas gerais [tese]. Belo Horizonte: Universidade Federal de Minas Gerais; 2012.

2O. Silva J A, Paula V S, Almeida A J, Villar L M. Investigação de acidentes biológicos entre profissionais de saúde. Esc Anna Nery Rev Enferm. 2009.

21. Reis PGTA, Driessen AL, Costa ACBA, Collaço IA, Tomasich FDS. Perfil epidemiológico de acidentes com material biológico entre estudantes de medicina em um pronto-socorro cirúrgico. Rev Col Bras Cir. 2013;4O(4):287-292.

22. Lima LM, Oliveira CC; Rodrigues KMR, Exposição ocupacional por material biológico no Hospital Santa Casa de Pelotas - 2004 a 2008. Esc Anna Nery Rev Enferm. 2011;15(1):96102.

23. Vieira M, Padilha MI, Pinheiro RDC. Análise dos acidentes com material biológico em trabalhadores da saúde. Rev. Lat Am. Enferm. 2011;19(2).

24. Gallas SR, Fontana RT. Biossegurança e a enfermagem nos cuidados clínicos: contribuições para a saúde do trabalhador. Rev Bras Enferm. 2010.63(5):786-92. 


\section{ANEXO - FICHA DE NOTIFICAÇÃO DO SINAN}

Republica Federativa do Brasil

Ministorio da Saúde

FICHA DE INVESTIOAÇĂO
SINAN

SISTEMA DE INFOREAÇC̄̃O DE AGRAVOS DE NOTHFCACĨO $\quad$ N ACIDENTE DE TRABALHO COM EXPOSIÇẢO Á MATERIAL BIOLÓGICO

Dofiniçấo de caso: Acidentes envolvendo sangue e outros fuidos orgáricos ocorídos com os profis sionais da área da saúde durante o desenvolvimento do seu trabalho, aonde os mesmos estâo expostos a materiais biológicos potencialmente contaminados,

Os ferimentos com aguhas e material perfuro cortante em geral sao considerados extremamente perigosos por serem potencialmente capazes de transmitir mais de 20 tipos de patogenos diferentes, sendo o virus da imunodeficiencia humana (HIV), o da hepatite B (HBV) o o da hepattite C (HCV) os agentes infocdosos mais comumente envolvidos.

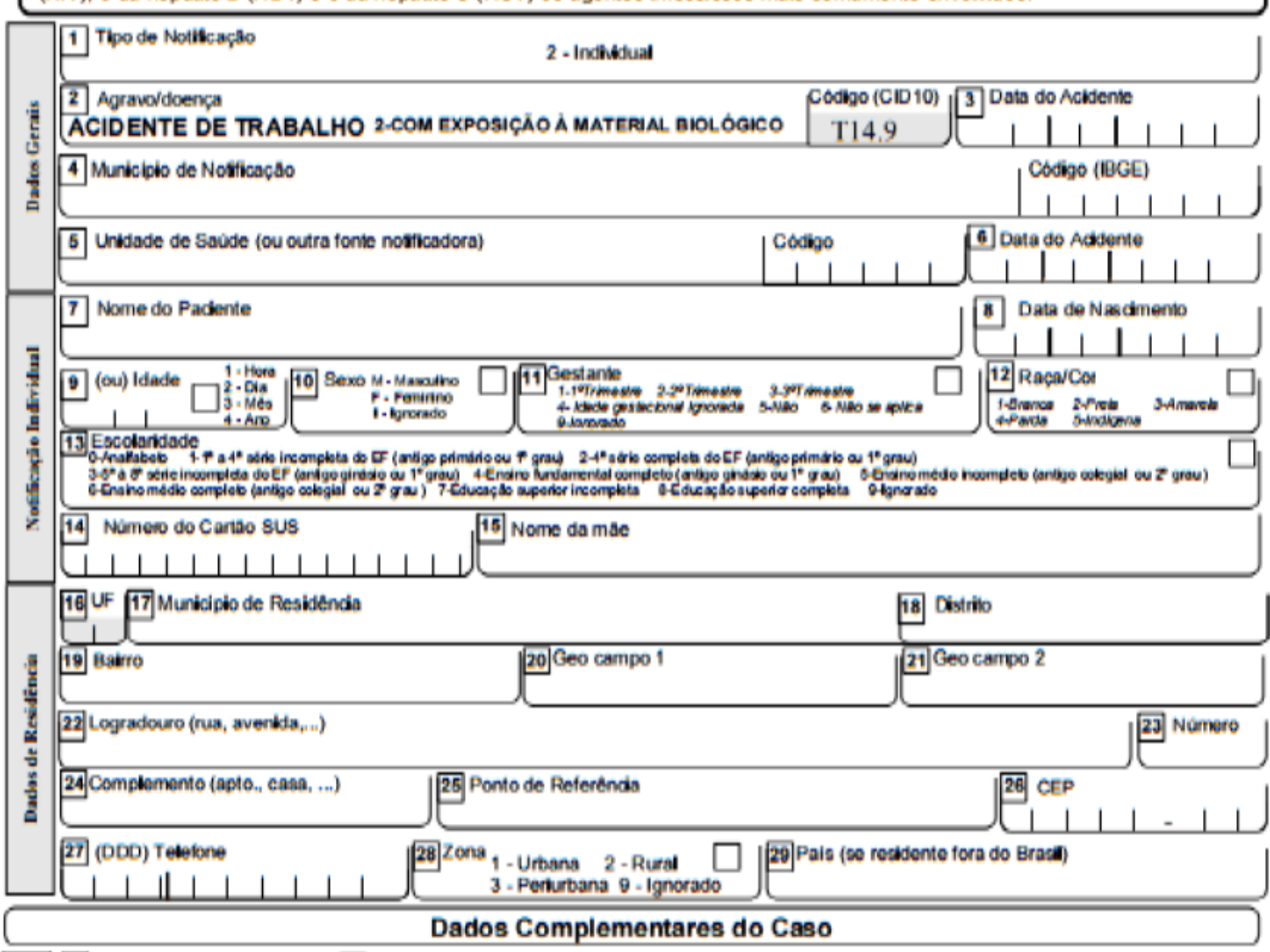

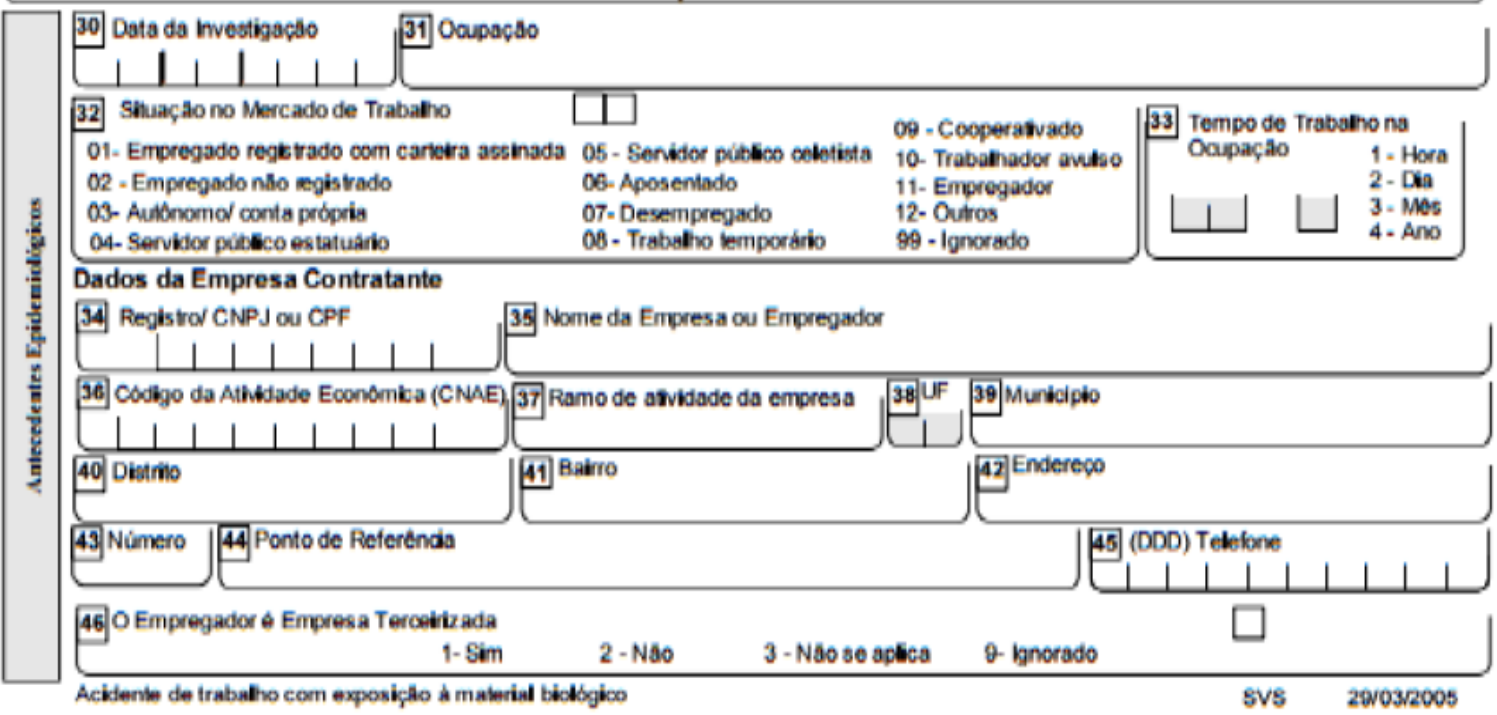




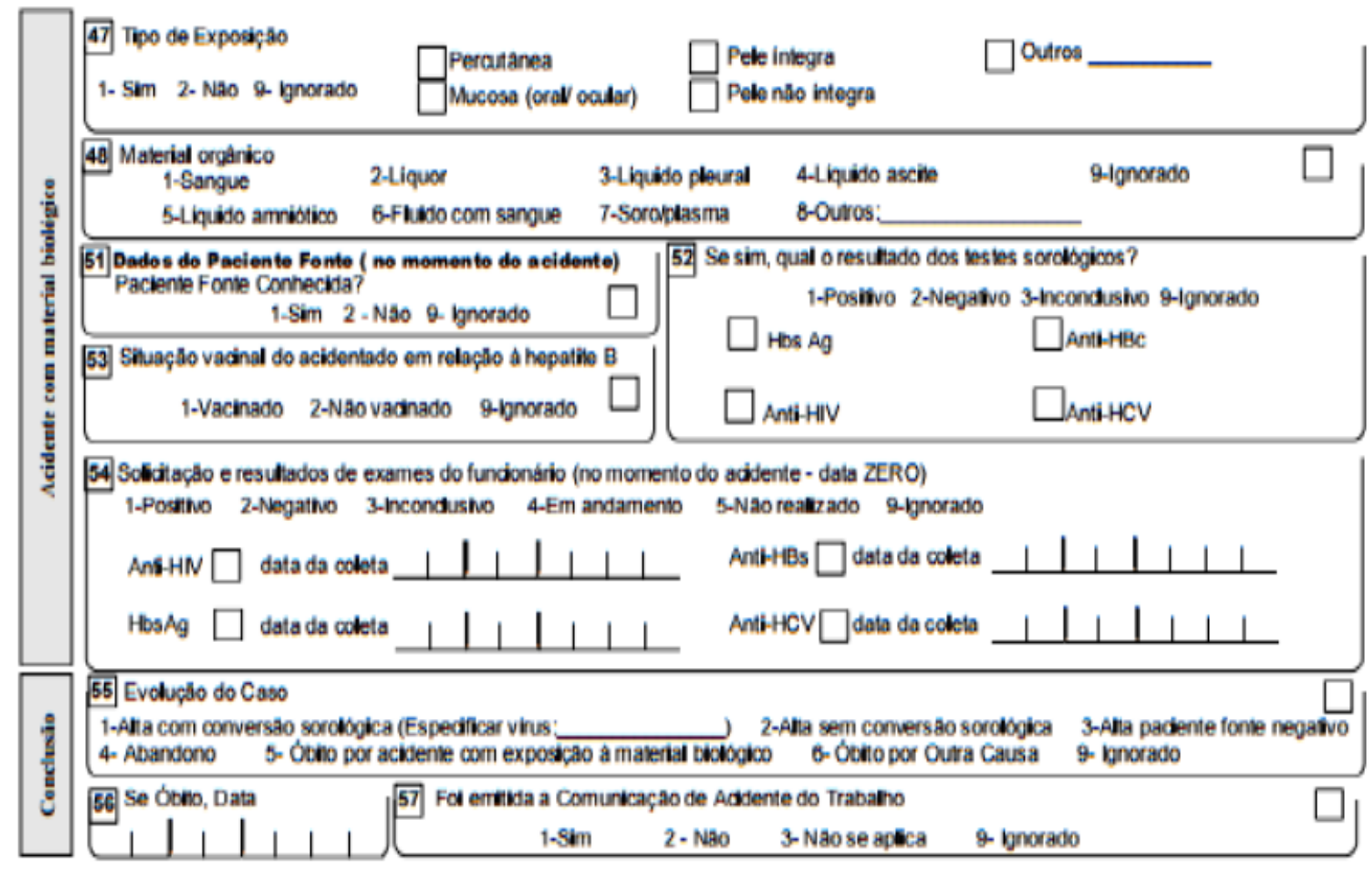

Informaçōes complementares e observaçōes

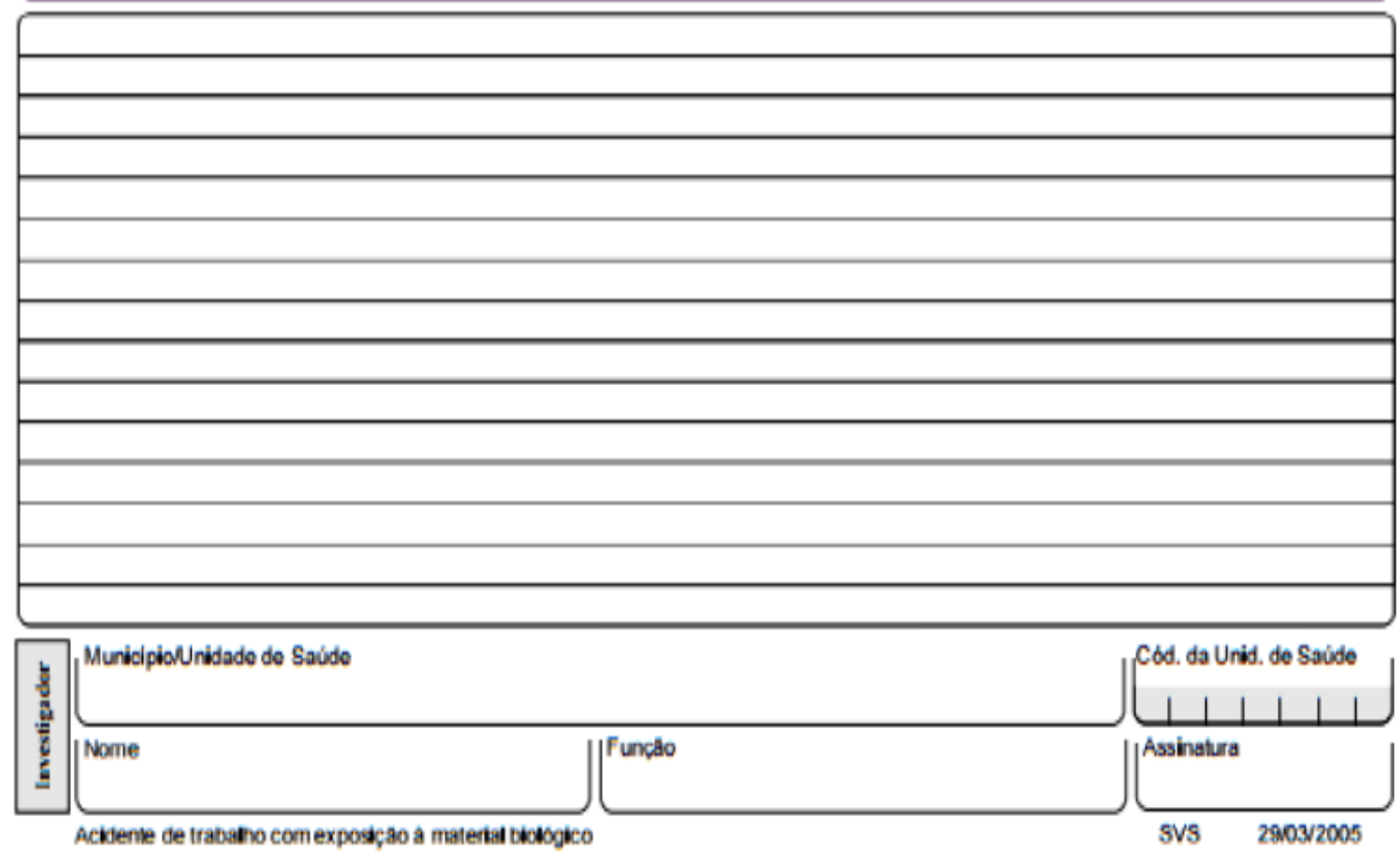

